\title{
Iron activated persulfate oxidation process for TMP degradation and kinetic analysis
}

\author{
H. ZHANG, B. LIANG, K. HAYES*
}

University of Michigan, Ann Arbor, MI 48109, USA

(*correspondence: ford@umich.edu)

Toxic pharmaceuticals residues found in aquatic environments pose a threat to human and ecosystem health. Recent studies show that reduced iron solid phases in aqueous systems may produce hydroxyl free radicals that can participate in transformation of aquatic organic contaminants. Furthermore, in the presence of peroxide or persulfate, these reduced iron solid phases generate highly reactive free radicals in higher concentrations that can completely remove those recalcitrant organic contaminants. In this study, different types of iron minerals have been applied to activate persulfate and degraded trimethoprim (TMP: a common pharmaceutical found in water). The results show that micron sulfidized zero-valent-iron (S-ZVI) is one of the most effective activators for persulfate activated oxidation of TMP. And the persulfate/S-ZVI oxidation system works well for a wide $\mathrm{pH}$ range of initial $\mathrm{pH}$ values, which eliminates the $\mathrm{pH}$ limitation for water treatment. A non-steady-state model is applied to predict the degradation kinetic of TMP by persulfate/S-ZVI oxidation system. Based on the results to date, the persulate/S-ZVI system shows promise for the complete and effective oxidation of pharmaceuticals and byproducts from water. 